\title{
Conexões
}

Artigo Original

\section{Influência do resultado momentâneo da partida na amplitude e profundidade da circulação da bola em pequenos jogos no futebol}

\author{
Arthur Souza De Vito ${ }^{1}$ \\ Juan Carlos Pérez Morales ${ }^{1}$ \\ Pablo Juan Greco ${ }^{1}$ \\ Gibson Moreira Praça ${ }^{1}$
}

\section{Resumo}

Objetivo: O objetivo do presente estudo foi comparar a amplitude e profundidade da circulação de bola das equipes em situações de vitória, empate e derrota durante a realização de pequenos jogos no futebol, em formato GR+3vs3+GR. Métodos: A amostra foi composta por 20 jogadores de futebol da categoria sub-17 de um clube de elite do Brasil. Avaliou-se a amplitude e profundidade da circulação de bola por meio das variáveis número de variações de corredor (NVC) e número de variações de profundidade (NVP), respectivamente. Relativizaram-se os valores de NVC e NVP com as três possíveis situações de resultado momentâneo (vitória, empate e derrota), por meio da razão entre os valores encontrados para ambas as variáveis e o tempo de posse de bola de cada equipe. Para comparar os valores nos três resultados parciais, recorreu-se à ANOVA one-way (NVC) e ao teste de Kruskall-Wallis (NVP). Resultados: Os resultados mostraram que houve diferenças significativas nos valores de NVC e NVP, com ambos sofrendo diminuição nas situações de vitória em comparação às situações de derrota. Conclusão: Conclui-se que o resultado momentâneo da partida influencia nos padrões da circulação de bola em pequenos jogos. Portanto, treinadores podem simular situações de vitória e derrota durante o treino, a fim de desencadear determinados comportamentos por parte dos jogadores.

Palavras-chave: Futebol. Educação física e Treinamento. Esportes.

\footnotetext{
${ }^{1}$ Universidade Federal de Minas Gerais, Escola de Educação Física, Departamento de Esportes, Belo Horizonte - MG, Brasil.

\section{Correspondência:}

Gibson Moreira Praça. Universidade Federal de Minas Gerais, Escola de Educação Física, Departamento de Esportes, Av. Pres. Antônio Carlos, 6627, CEP 31270-901, Pampulha, Belo Horizonte - MG, Brasil, Email: gibson moreira@yahoo.com.br
} 


\section{The influence of match status on the width and depth of ball circulation in small-sided games in soccer}

\section{ABSTRACT}

Objective: This study aimed to compare the width and depth of the teams' ball circulation in situations of winning, drawing and losing in soccer small-sided games in GR + 3vs $3+$ GR format. Methods: The sample consisted of $20 \mathrm{U}-17$ soccer players from a Brazilian elite club. The width and depth of the ball circulation were evaluated through the variables NVC (number of corridor variations) and NVP (number of depth variation), respectively. The values of NVC and NVP were compared between the three possible situations of momentary outcome (winning, drawing and losing), by the ratio between the values found for both variables and the time of possession of each team's ball. To compare the values in the three partial results, one-way ANOVA (NVC) and the Kruskall-Wallis test (NVP) were used. Results: The results showed that there were significant differences in NVC and NVP values, with both suffering a decrease in winning situations compared to losing situations. Conclusion: It is concluded that the momentary result of the match influences the ball circulation patterns in small-sided games. Therefore, coaches can simulate situations of winning and losing during the training in order to trigger certain behaviors on the part of the players.

Keywords: Soccer. Physical Education and Training. Sports.

\section{La influência del resultado momentáneo en la amplitude y profundidad de la circulación de la pelota em pequeños juegos de fútbol}

\section{RESUMEN}

Objetivo: Se comparó la amplitud y profundidad de la circulación del balón de 20 jugadores de fútbol de la categoría sub-17 de um club de elite durante la realización de pequeños juegos en la situación de 3 contra 3 más el guardameta, en condiciones de victoria, empate y derrota. Métodos: Se evaluó la amplitud y profundidad de la circulación de balón por medio del número de variaciones de pasillo (NVPa) y números de variaciones de profundidad (NVPro), respectivamente. Se relativizaron los valores de NVPa y NVPro con las tres posibles situaciones de resultado momentáneo (victoria, empate y derrota), por medio de la razón entre los valores encontrados para ambas variables y el tiempo de posesión de balón de cada equipo. Para comparar los valores en los tres resultados parciales, se recurrió a la ANOVA one-way para NVPa y la prueba de Kruskall-Wallis para NVPro. Resultados: Los resultados indicaron diferencias significativas en los valores de NVPa y NVPro con disminución en las situaciones de victoria en comparación con las situaciones de derrota. Conclusión: Se concluye que el resultado momentáneo del juego influye en los patrones de la circulación del balón en pequeños juegos. Por lo tanto, los entrenadores pueden simular situaciones de victoria y derrota para desencadenar determinados comportamientos por parte de los jugadores.

Palabras Clave: Fútbol. Educación física y formación. Deportes. 


\section{INTRODUÇÃO}

O futebol, integrado ao grupo dos Jogos Esportivos Coletivos (JEC), caracteriza-se como um esporte de invasão, no qual se observam situações de oposição e cooperação entre os jogadores e em que as ações de ataque e defesa ocorrem de maneira simultânea (GARGANTA; GRÉHAIGNE, 1999). Neste contexto, o desempenho no futebol é multifatorial, envolvendo a interação entre componentes físicos, técnicos, psicológicos e táticos (AGUIAR et al., 2012). De forma a contemplar estas características do jogo, propõe-se na literatura a utilização de pequenos jogos como meio de treino no futebol, uma vez que estes permitem os estímulos de ordem fisiológica, técnica e tática simultaneamente (AGUIAR et al., 2012; HILL-HAAS et al., 2008; PAMPININI, 2007), em contexto decisional similar ao jogo formal - ataque, defesa, cooperação e oposição.

Recentemente, investigou-se a influência de variáveis situacionais no comportamento individual dos jogadores e de equipes durante o jogo (LAGO, 2009). Um destes fatores diz respeito ao resultado momentâneo da partida (MORAES; CARDOSO; TEOLDO, 2014), entendido como o resultado momentâneo do jogo (empate, derrota ou vitória). Na literatura, sugere-se a existência de diferenças nos comportamentos individuais e coletivos de uma equipe a partir da modificação deste resultado momentâneo da partida (CARVALHO; SCAGLIA; TEOLDO, 2013), como diferentes durações da posse de bola e os locais onde as ações acontecem. Tal fato ocorre devido à necessidade de uma equipe em se adaptar e readaptar perante as variações do placar durante uma partida (MORAES; CARDOSO; TEOLDO, 2014), pressupondo que esta tentará sempre evitar um revés ou buscar a vitória. Apesar de Carvalho et al. (2013) terem verificado a influência do desempenho tático no resultado final de pequenos jogos, até o presente momento não foi verificada a influência do resultado momentâneo da partida nas ações desempenhadas pelos jogadores nos pequenos jogos. Entende-se que o resultado momentâneo do jogo se apresente como variável potencialmente manipulável em pequenos jogos (i.e., simular situações em que determinada equipe começa com resultado favorável ou contrário durante o pequeno jogo). Contudo, desconhece-se o efeito que este resultado apresenta no comportamento dos jogadores.

O sucesso no futebol é influenciado por diversas variáveis, sendo a circulação de bola um dos preditores deste sucesso (LAGO-PEÑAS; LAGO-BALLESTEROS; REY, 2011). Entende-se a circulação da bola como uma sucessão de passes e/ou conduções visando a progressão até a área adversária ou a manutenção da posse da bola (GARGANTA, 1997). Esta circulação baseia-se nos princípios de jogo em amplitude, que corresponde à distância máxima entre jogadores da mesma equipe no que diz respeito à largura do campo de jogo; e profundidade que, por sua vez, refere-se à distância entre o jogador mais próximo da própria baliza e o mais próximo à baliza adversária (FOLGADO et al., 2014). Barreira et al. (2011) observaram as partidas referentes aos play-offs da EURO 2008 e encontraram que 
as equipes, em situações adversas no placar, tendiam a possuir um maior tempo de posse de bola em zonas mais avançadas do campo, sugerindo assim que estas equipes apresentaram maior profundidade no campo de jogo. Contudo, nenhum estudo investigou, até o momento, os padrões de circulação em amplitude e profundidade em situações de pequenos jogos, os quais também não foram investigados quanto à influência do resultado momentâneo do jogo. Portanto, o objetivo do presente estudo foi comparar a amplitude e profundidade da circulação de bola das equipes em situações de vitória, empate e derrota durante a realização de pequenos jogos no futebol, em formato GR+3vs3+GR. Dada a similaridade contextual do pequeno jogo em relação ao jogo formal, hipotetiza-se que as equipes em desvantagem no placar, durante pequenos jogos, buscarão uma maior circulação de bola visando superar a defesa adversária para que, desta forma, marquem os gols necessários para igualar o placar.

\section{MÉTODO}

\section{AMOSTRA}

Participaram do estudo 20 jogadores de futebol (idade: 16,2 $\pm 0,9$ anos), pertencentes à categoria sub-17 de um clube de nível nacional, situado na cidade de Belo Horizonte. Todos os atletas tinham no mínimo cinco anos de prática deliberada da modalidade. O estudo foi aprovado pelo comitê de ética da Universidade Federal de Minas Gerais - COEP, sob o CAE 510119.9.0000.5149. Os participantes e seus responsáveis legais assinaram um termo de assentimento e consentimento livre e esclarecido, respectivamente.

\section{Procedimentos}

A composição das equipes baseou-se na capacidade tática e na posição dos jogadores de forma a gerar confrontos equilibrados, conforme recomendado na literatura (FOLGADO et al., 2014). Dessa forma, cada equipe foi composta por um goleiro, um defensor, um meio campista e um atacante. Para a mensuração da capacidade tática, utilizada para balancear as equipes, os atletas, com exceção dos goleiros, foram submetidos ao teste de campo do Sistema de Avaliação Tática no Futebol - FUT-SAT (TEOLDO et al., 2011), realizado no primeiro dia de coletas. O teste consistiu em um jogo no formato de GR+3vs3+GR, em um campo de $36 \mathrm{~m}$ x 27m, com duração de 4 minutos, com a manutenção de todas as regras do jogo formal. Para esse procedimento, os atletas foram divididos em três grupos de mesmo estatuto posicional, os quais realizaram um jogo entre si (atacantes $x$ defensores, por exemplo). Por meio do percentual de acerto dos princípios táticos, avaliado a posteriori por peritos, foram selecionados os três atletas de cada estatuto posicional que obtiveram melhor desempenho, para compor o grupo 1 (equipes $A, B$ e $C$ ) e os três atletas dos diferentes estatutos posicionais com pior desempenho, para compor o grupo 2 (equipes D, E e F). Dessa forma, os atletas 
com maior nível tático não enfrentaram equipes com atletas de nível tático inferior, reduzindo a influência da modificação do nível do adversário no comportamento dos atletas durante o pequeno jogo (PRAÇA, 2017).

Após os testes, os atletas participaram da segunda etapa do estudo, referente à realização dos pequenos jogos. As sessões de coleta de dados referentes aos pequenos jogos ocorreram durante 3 semanas, sendo todos os jogos realizados no mesmo horário do dia. Todas as equipes realizaram o mesmo número de jogos, de forma a contemplar todos os confrontos possíveis dentro de um mesmo grupo. Os jogos ocorreram no formato de GR+3vs3+GR, no espaço de $36 \times 27$ metros, que corresponde à dimensão utilizada no teste de campo do Sistema de Avaliação Tática no Futebol - FUT-SAT (TEOLDO et al., 2011). Desta forma, ocorreram 4 dias de coleta, conforme explicado na Figura 1. Cada sessão de coleta teve início com uma atividade preparatória, com duração de 10 minutos. A atividade preparatória seguiu um rotina padronizada no clube, composta inicialmente por deslocamentos sem bola (com mudança de direção e de velocidade) e, na sequência, por ações técnicas com bola (individuais e em dupla). Para otimizar o tempo e espaço físico necessários para a coleta, ocorreram dois confrontos de cada equipe em cada dia de coleta, de forma alternada, conforme demonstra a Figura 2.

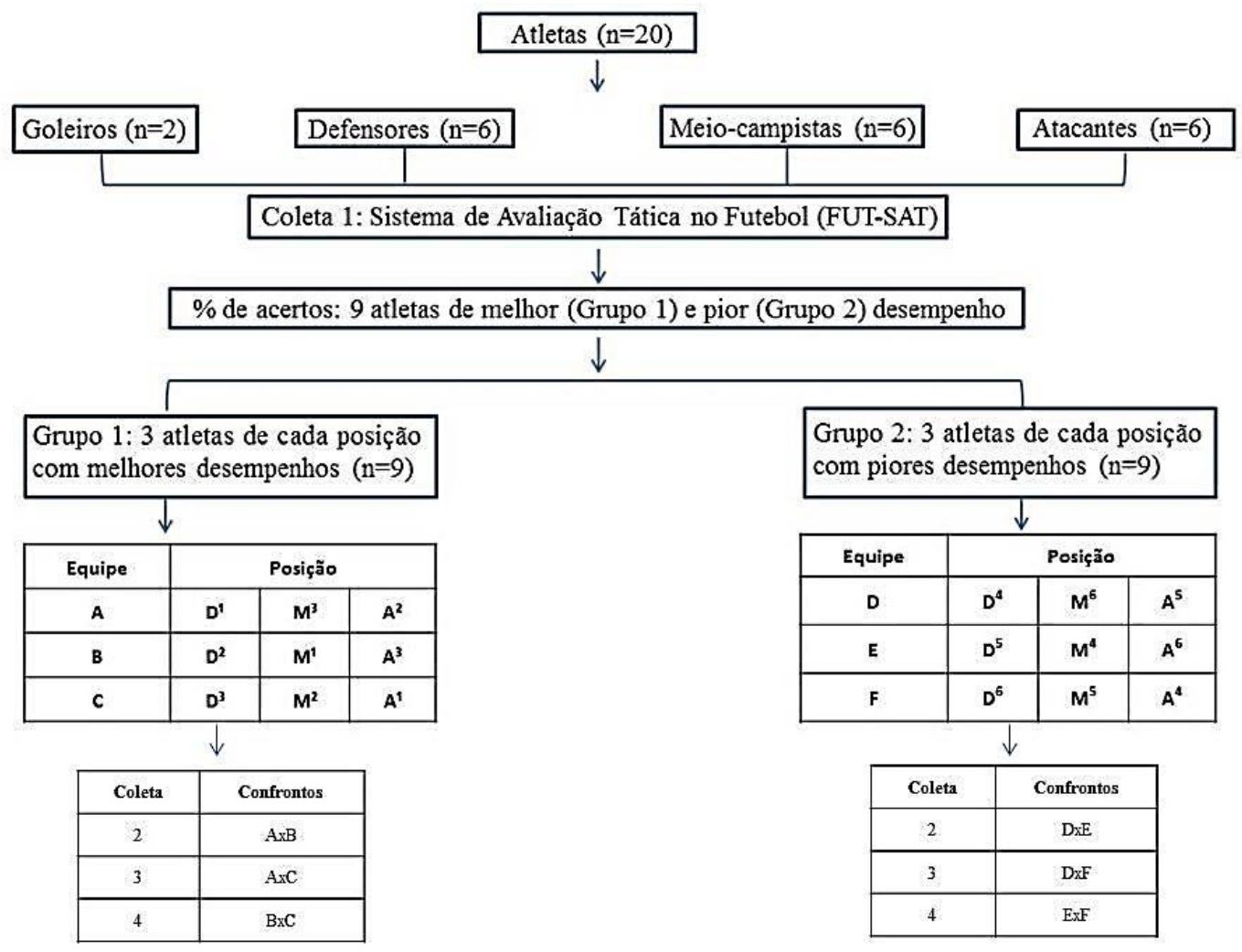

Legenda: Posições: $D=$ Defensor; $M=$ Meio-campista; $A=$ Atacante. Números sobrescritos indicam o ranking, relativo à posição, que o jogador obteve no teste utilizado para a composição das equipes do maior (1) até o menor (6).

Figura 1 - Procedimentos realizados no estudo. 


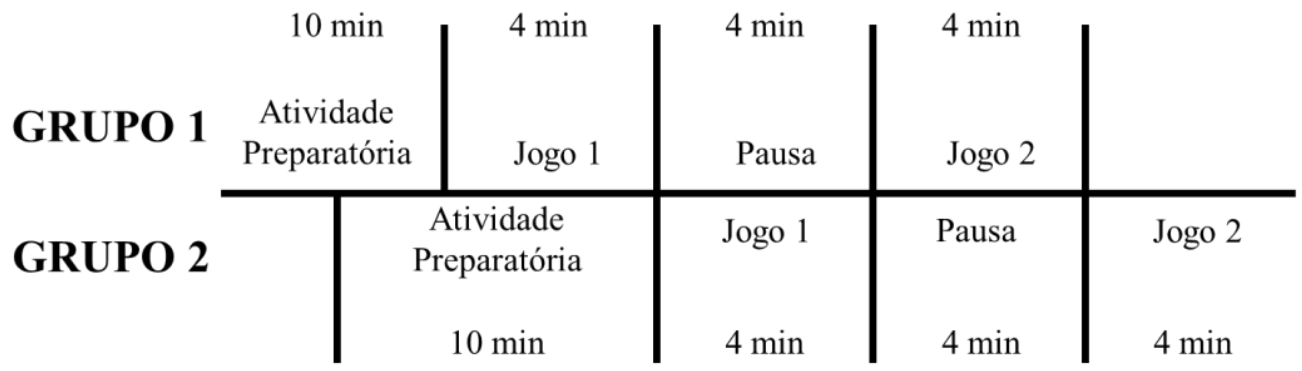

Figura 2 - Descrição do dia da coleta.

\section{INSTRUMENTOS}

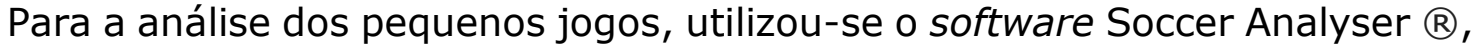
o qual permite a divisão do campo em corredores laterais (Figura 3) e meio-campo ofensivo e defensivo (Figura 4).

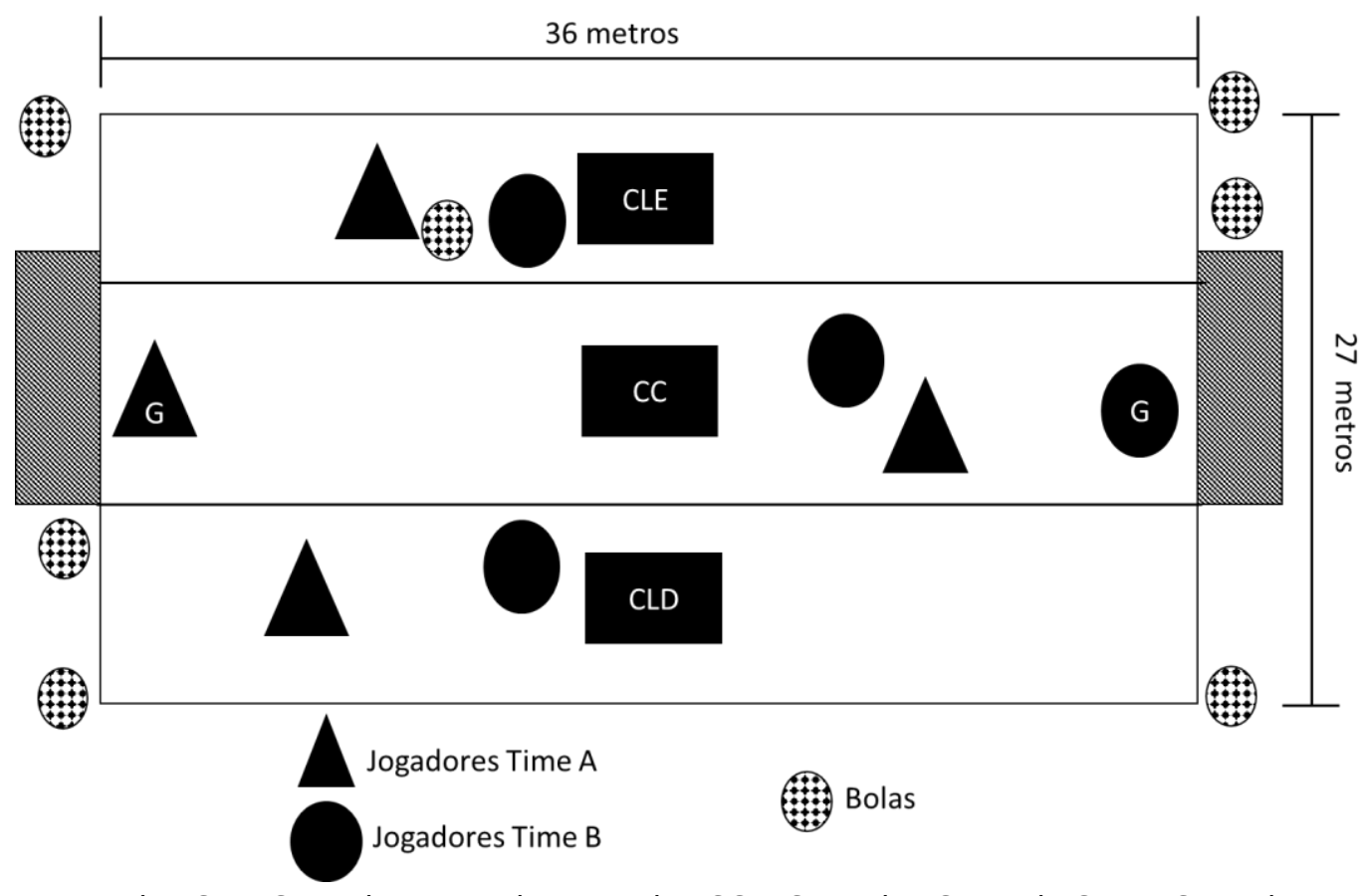

Legenda: $\mathrm{CE}=$ Corredor Lateral Esquerdo; $\mathrm{CC}=$ Corredor Central; $\mathrm{CLD}=$ Corredor Lateral Direito.

Figura 3 - Divisão do campo em corredores (amplitude). 


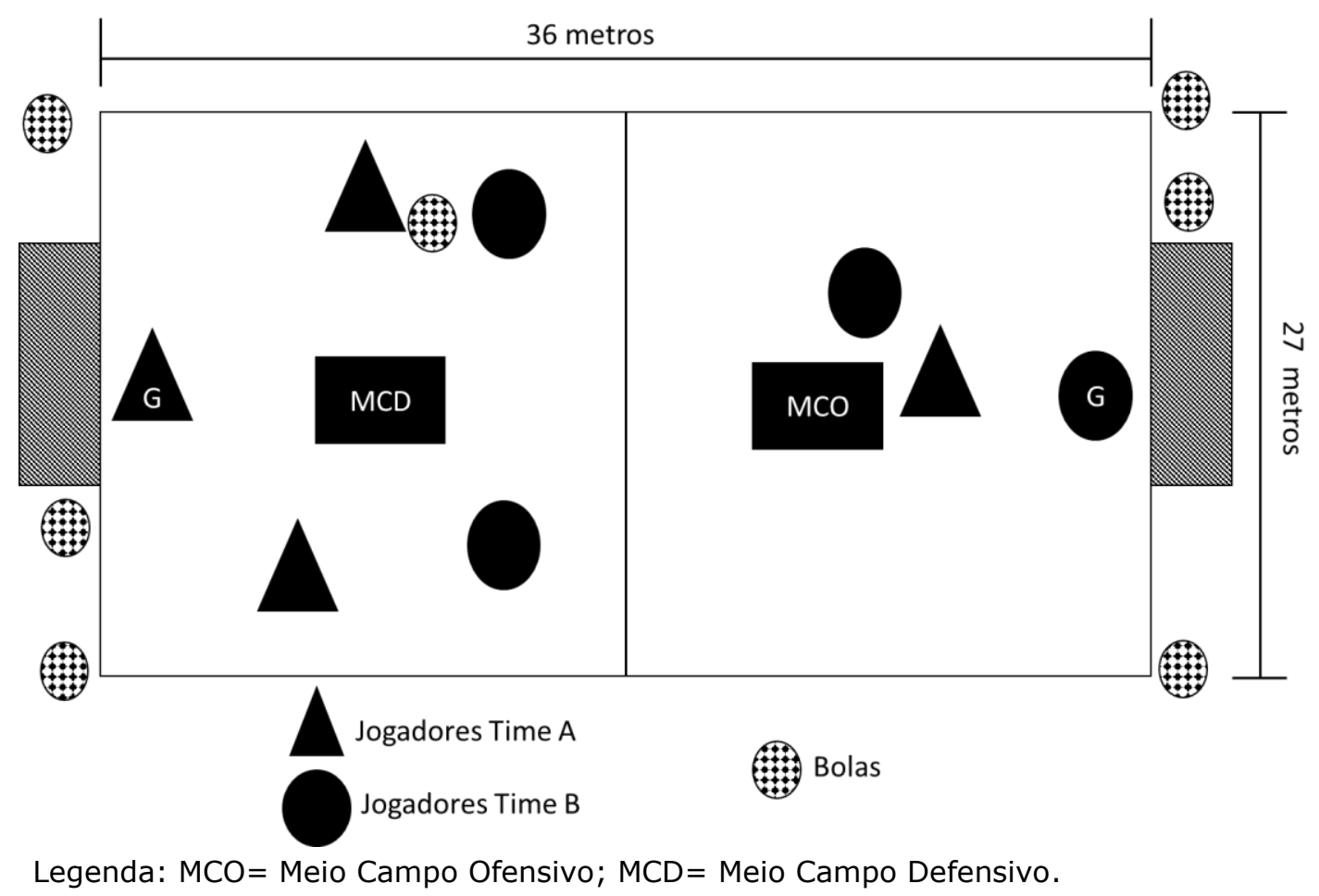

Figura 4 - Divisão do campo em profundidade.

No intuito de avaliar a amplitude da circulação da bola no jogo, utilizou-se a variável "número de variação de corredor" (NVC), que diz respeito ao número de vezes que a bola circula de um corredor ao outro, seja por meio de passe ou condução (GARGANTA, 1997). Para mensurar a profundidade, registrou-se o número de vezes em que a equipe circulou a bola, por meio de passe ou condução de bola, do meio campo defensivo (MCD) ao meio campo ofensivo (MCO), resultando em um "número de variação de profundidade" (NVP). Em ambos os casos, foram consideradas apenas variações de corredor/profundidade bemsucedidas, isto é, se a bola se manteve em posse da equipe no novo local do campo (seja pela realização de um passe positivo, seja pela condução da bola). Por fim, para relativizar os valores de NVC e NVP com as três possíveis situações relacionadas ao resultado momentâneo da partida (perdendo, empatando e vencendo), foi feita a razão entre o NVC e NVP e o tempo em que as equipes obtinham a posse de bola em cada uma das situações, excluindo-se o tempo de bola parada (bola fora do campo de jogo ou falta), obtendo assim valores de $\mathrm{NVC} /$ minuto e NVP/minuto para cada resultado parcial da partida.

\section{ANÁLISE DE DADOS}

Inicialmente foram verificados os pressupostos de normalidade (ShapiroWilk) e homocedasticidade (teste de Levene). Para a variável NVC, atendidos os pressupostos, recorreu-se à ANOVA one-way para a comparação dos valores nos três resultados parciais. Já para a variável NVP, na medida em que o pressuposto de normalidade não foi atendido, recorreu-se ao teste de Kruskall-Wallis para a comparação dos valores. Calculou-se ainda o tamanho do efeito $\eta^{2}{ }^{2}$, classificado 
em pequeno (0.01), moderado (0.06) e grande (0.14) (COHEN, 1988). Em todos os casos, adotou-se o nível de significância de $5 \%$. Utilizou-se o software SPSS 20.0 para as análises.

\section{Resultados E Discussão}

A Tabela 1 apresenta os valores médios, desvios padrão e p-valor do Número de Variação de Corredores (NVC) e Número de Variações de Profundidade (NVP) em função dos três momentos relacionados ao resultado momentâneo da partida (empate, derrota e vitória).

Tabela 1 - Número de variações de corredores e profundidade em cada resultado momentâneo

\begin{tabular}{lcc}
\hline \multicolumn{1}{c}{ Momento } & NVC & NVP \\
\hline Empate $^{1}$ & $13,04(5,83)$ & $5,45(2,46)$ \\
Derrota $^{2}$ & $13,63(5,39)^{*}$ & $7,00(5,70)^{*}$ \\
Vitória $^{3}$ & $10,00(11,05)$ & $5,00(4,86)$ \\
p-valor & 0,009 & 0,018 \\
Tamanho do efeito & 0,256 & 0,222 \\
Comparações pareadas & $3<2$ & $3<2$ \\
\hline * Diferenças significativas & &
\end{tabular}

Os resultados demonstram que houve diferenças significativas nos valores de NVC e NVP para as situações de vitória e derrota, observando-se menores valores de NVP ( $p=0,018$; efeito grande) e NVC ( $p=0,009$; efeito grande) nas situações de vitória em comparação às situações de derrota. Reportou-se, ainda, tamanho do efeito grande para as duas análises, indicando elevada magnitude da diferença entre os valores.

A investigação sobre o comportamento dos jogadores durante pequenos jogos no futebol busca fornecer informações para treinadores sobre a influência da tarefa e do ambiente no comportamento dos atletas. Contudo, poucos estudos, até o presente momento, investigaram a influência de variáveis situacionais no comportamento de atletas durante o treino no futebol. Neste cenário, o presente estudo objetivou comparar a amplitude e profundidade da circulação de bola das equipes nas situações de vitória, empate e derrota na prática de pequenos jogos no futebol. Os resultados demonstraram que o comportamento dos jogadores sofreu influência do resultado momentâneo da partida, apresentando valores significativamente maiores de NVC e NVP em situações de derrota, se comparados aos momentos de vitória das equipes.

Estudos demonstraram que mudanças de comportamento relacionadas à posse de bola foram ocasionadas pelos diferentes resultados momentâneos da partida em jogos formais (LAGO, 2009; LAGO-PEÑAS, DALLAL, 2010). 
Especificamente, observa-se que situações de derrota elevam a frequência de passes curtos (KONEFAŁ et al., 2018) e longos (PAIXÃO et al., 2015) dos times em jogos formais. De maneira geral, justifica-se o aumento na incidência de passes pela necessidade de circular a bola em razão da necessidade de enfrentar defesas em bloco baixo das equipes em vitória (PRAÇA et al., 2019). No presente estudo, equipes em situação de derrota apresentaram maior incidência de variações de corredor e profundidade em comparação aos times em situação de vitória. Entende-se que o aumento no número de variações de corredor e de profundidade represente uma maior circulação da bola durante o momento de organização ofensiva, o que está em consonância com estudos publicados nos jogos formais. Neste sentido, sugere-se que o pequeno jogo 3vs.3 represente dinâmicas semelhantes ao jogo formal no que se refere à influência do resultado momentâneo da partida no comportamento dos jogadores.

Considerando a escassez de informações a respeito da influência de variáveis situacionais no comportamento dos jogadores durante os pequenos jogos no futebol, sugerem-se possíveis implicações práticas dos resultados do presente estudo no auxílio a treinadores e pesquisadores para uma melhor condução do processo de ensino-aprendizagem-treinamento na modalidade. Particularmente, recomenda-se que treinadores tenham em mente que o mesmo pequeno jogo pode apresentar implicações diferentes para os jogadores caso, por exemplo, uma equipe marque um gol no início e consiga permanecer à frente no placar durante a maior parte da série. Assim, de forma a permitir adequada vivência da maior amplitude possível de situações-problema, treinadores devem ajustar confrontos durante séries de $\mathrm{PJ}$ de forma a permitir maior variabilidade no resultado momentâneo. Contudo, sugerem-se novos estudos envolvendo outras configurações de pequenos jogos - por exemplo, um maior número de jogadores - para verificar se resultados similares serão observados.

Este estudo, embora seja o primeiro a investigar a influência de variáveis situacionais no comportamento dos jogadores, apresenta limitações que devem ser levadas em conta. Embora o uso de pequenos jogos apresente respaldo da literatura para o aprimoramento de capacidades táticas no futebol (PRÁXEDES et al., 2019), sugere-se que a resposta dos jogadores dependa das configurações do jogo (por exemplo com modificações no número de jogadores e nas dimensões do campo), Desta forma, não se pode afirmar que tais mudanças de comportamento estarão presentes na mesma magnitude durante outros formatos de pequenos jogos. Neste ponto, sugere-se a condução de novos estudos que contemplem diferentes configurações de pequenos jogos. Além disso, a realização das coletas contemplando apenas uma categoria (sub-17) pode não ser capaz de descrever o comportamento de atletas pertencentes a categorias inferiores ou superiores. Portanto, sugere-se que investigações considerem diferentes categorias, visando um melhor entendimento sobre como o resultado momentâneo influencia o comportamento de jogadores de diversas categorias. Por fim, considerando que a utilização do pequeno jogo assenta-se numa perspectiva de integração dos 
estímulos táticos, técnicos, físicos e psicológicos durante o processo de treinamento no futebol (SARMENTO et al., 2018), sugere-se que futuros estudos incluam variáveis dependentes de ordem técnica, física e fisiológica para o completo entendimento da influência do resultado momentâneo no comportamento dos jogadores durante pequenos jogos.

\section{CONSIDERAÇÕES FINAIS}

Conclui-se que o resultado momentâneo da partida influencia o padrão de circulação da bola durante o pequeno jogo 3vs.3 no futebol. Especificamente, o resultado de derrota induz a um comportamento de circulação da bola na busca por espaços defensivos, ao passo que a situação de vitória induz a uma ação mais direta, com menor circulação, dos jogadores em posse da bola.

\section{REFERÊNCIAS}

AGUIAR, Marco; BOTELHO, Goretti; LAGO, Carlos; MAÇAS, Victor; SAMPAIO, Jaime. A review on the effects of soccer small-sided games. Journal of Human Kinetics, v. 33, p. 103-113, 2012. Disponível em:

https://www.ncbi.nlm.nih.gov/pmc/articles/PMC3588672/.

BARREIRA, Daniel; GARGANTA, Júlio; ANGUERA, Teresa. In search of nexus between attacking game-patterns, match status and type of ball recovery in European Soccer Championship 2008. Research Methods and Performance Analysis, v. 226, p. 226-237, 2011. Disponível em:

https://www.researchgate.net/publication/260192349 In search of nexus between att acking game-

patterns match status and type of ball recovery in European Soccer Championship $\underline{2008}$.

COHEN, Jacob. Statistical power analysis for the behavioral sciences. $2^{\text {nd }}$. Nova Iorque: Lawrence Erlbaum Associates, 1988.

FOLGADO, Hugo. DUARTE, Ricardo; FERNANDES, Orlando; SAMPAIO, Jaime. Competing with lower level opponents decreases intra-team movement synchronization and timemotion demands during pre-season soccer matches. PloS one, v. 9, n. 5, p. e97145, 2014. Disponível em:

https://journals.plos.org/plosone/article?id=10.1371/journal. pone.0097145.

FOLGADO, Hugo; LEMMINK, Koen A. P. M.; FRENCKEN, Wouter; SAMPAIO, Jaime.

Length, width and centroid distance as measures of teams' tactical performance in youth football. European Journal of Sport Science, v. 14, n. sup1, p. S487-S492, 2014.

Disponível em: https://www.ncbi.nlm.nih.gov/pubmed/24444244.

GARGANTA, Júlio. Modelação táctica do jogo de Futebol: Estudo da organização da fase ofensiva em equipas de alto rendimento. 1997. (Doutorado). Faculdade de Ciências do Desporto, Universidade do Porto, 1997. 
GARGANTA, Júlio; GRÉHAIGNE, Jean F. Abordagem sistêmica do jogo de futebol: moda ou necessidade?. Movimento, Porto Alegre, v. 5, n. 10, p. 40-50, 1999. Disponível em: https://seer.ufrgs.br/Movimento/article/view/2457.

HILL-HAAS, Stephen V.; COUTTS, Aaron J.; ROWSELL, Greg; DAWSON, Brian. Variability of acute physiological responses and performance profiles of youth soccer players in small-sided games. Journal of Science and Medicine in Sport, v. 11, n. 5, p. 487-490, 2008. Disponível em: https://www.ncbi.nlm.nih.gov/pubmed/17825620.

KONEFA $Ł$, Marek; CHMURA, Paweł; ZACHARKO, Michał; CHMURA, Jan; ROKITA, Andrzej; ANDRZEJEWSKI, Marcin. Match outcome vs match status and frequency of selected technical activities of soccer players during UEFA Euro 2016. International Journal of Performance Analysis in Sport, v. 18, n. 4, p. 568-581, 2018. Disponível em: https://www.tandfonline.com/doi/full/10.1080/24748668.2018.1501991.

LAGO-PEÑAS, Carlos. The influence of match location, quality of opposition, and match status on possession strategies in professional association football. Journal of Sports Sciences, v. 27, n. 13, p. 1463-1469, 2009. Disponível em: https://www.ncbi.nlm.nih.gov/pubmed/19757296.

LAGO-PEÑAS, Carlos; DELLAL, Alexandre. Ball possession strategies in elite soccer according to the evolution of the match-score: the influence of situational variables. Journal of Human Kinetics, v. 25, p. 93-100, 2010. Disponível em: https://content.sciendo.com/view/journals/hukin/25/1/article-p93.xml.

LAGO-PEÑAS, Carlos; MARTÍN, Rafael. Determinants of possession of the ball in soccer. Journal of Sports Sciences, v. 25, n. 9, p. 969-974, 2007. Disponível em: https://www.ncbi.nlm.nih.gov/pubmed/17497397.

LAGO-PEÑAS, Carlos; LAGO-BALLESTEROS, Joaquín; REY, Ezequiel. Differences in performance indicators between winning and losing teams in the UEFA Champions League. Journal of Human Kinetics, v. 27, p. 135-146, 2011. Disponível em: https://content.sciendo.com/view/journals/hukin/27/1/article-p135.xml.

CARVALHO, Felipe Moniz; SCAGLIA, Alcides J.; TEOLDO, Israel C. Influência do desempenho tático sobre o resultado final em jogo reduzido de futebol. Revista da Educação Física/UEM, Maringá, v. 24, n.3, p. 393-400. 2013. Disponível em: http://www.periodicos.uem.br/ojs/index.php/RevEducFis/article/view/18902/0.

MORAES, Emerson L.; CARDOSO, Felippe; TEOLDO, Israel C. Análise dos padrões ofensivos da Seleção Espanhola de Futebol na Copa do Mundo FIFA® 2010 em relação ao" status" da partida. Revista Brasileira de Educação Física e Esporte, v. 28, n. 3, p. 361-369, 2014. Disponível em: http://www.scielo.br/scielo.php?pid=S1807$\underline{5092014000300361 \& \text { script }=\text { sci abstract\&tlng=pt. }}$

PAIXÃO, Paulo; SAMPAIO, Jaime; ALMEIDA, Carlos H.; DUARTE, Ricardo. How does match status affects the passing sequences of top-level European soccer teams?. International Journal of Performance Analysis in Sport, v. 15, n. 1, p. 229-240, 2015. Disponível em: https://www.tandfonline.com/doi/abs/10.1080/24748668.2015.11868789.

PRAÇA, Gibson M.; MORALES, Juan C. P.; MOREIRA, Pedro E. D.; PEIXOTO, Gustavo H. C.; BREDT, Sara T.; CHAGAS, Mauro H.; TEOLDO, Israel C.; GRECO, Pablo J. Tactical behavior in soccer small-sided games: influence of team composition criteria. Revista Brasileira de Cineantropometria \& Desempenho Humano, v. 19, n. 3, p. 354-363, 2017. Disponível em: http://www.scielo.br/scielo.php?script=sci arttext\&pid=S198000372017000300354. 
PRAÇA, Gibson M.; LIMA, Bernardo B.; BREDT, Sara T.; SOUSA, Raphael B.; CLEMENTE, Filipe M.; ANDRADE, André G. Influence of match status on players' prominence and teams' network properties during 2018 FIFA World Cup knockout phase. Frontiers in Psychology, v. 10, p. 695, 2019. Disponível em:

https://www.frontiersin.org/articles/10.3389/fpsyg.2019.00695/full.

PRÁXEDES, Alba; DEL VILLAR ÁLVAREZ, Fernando; MORENO, Alberto; GIL-ARIAS, Alexander; DAVIDS, Keith. Effects of a nonlinear pedagogy intervention programme on the emergent tactical behaviours of youth footballers. Physical Education and Sport Pedagogy, p. 1-12, 2019. Disponível em: https://www.tandfonline.com/doi/full/10.1080/17408989.2019.1580689.

RAMPININI, Ermanno; IMPELLIZZERI, Franco M.; CASTAGNA, Carlos; GRANT, Abt; CHAMARI, Karim; SASSI, Aldo; MARCORA, Samuele M. Factors influencing physiological responses to small-sided soccer games. Journal of Sports Sciences, v. 25, n. 6, p. 659666, 2007. Disponível em: https://www.ncbi.nlm.nih.gov/pubmed/17454533.

SARMENTO, Hugo; CLEMENTE, Filipe M.; HARPER, Liam D.; TEOLDO, Israel C.; OWEN, Adam; FIGUEIREDO, António J. Small sided games in soccer-a systematic review. International Journal of Performance Analysis in Sport, v. 18, n. 5, p. 693-749, 2018. Disponível em: https://www.tandfonline.com/doi/full/10.1080/24748668.2018.1517288.

TEOLDO, Israel C.; GARGANTA, Júlio; GRECO, Pablo J.; MESQUITA, Isabel; MAIA, José. Sistema de avaliação táctica no Futebol (FUT-SAT): Desenvolvimento e validação preliminar. Motricidade, v. 7, n. 1, p. 69-84, 2011. Disponível em: http://www.scielo.mec.pt/scielo.php?script=sci arttext\&pid=S1646107X2011000100008. 